\title{
Seasonal variations in the intensity of muon bundles detected at the ground level
}

\author{
R.P. Kokoulin ", A.G. Bogdanov, A.N. Dmitrieva, E.A. Kovylyaeva, \\ E.V. Romanenkova, V.V. Shutenko \\ National Research Nuclear University MEPhI (Moscow Engineering Physics Institute), \\ Moscow 115409, Russian Federation \\ E-mail: rpkokoulin@mephi.ru
}

\begin{abstract}
Experimental data accumulated in a 3-year long series of measurements (from May 2012 to April 2015) of cosmic ray muon bundles with the coordinate-tracking detector DECOR are analyzed. It has been found that the measured rate of the events exhibits clear seasonal variations, repeated every year of observations: in winter, the event rate is significantly higher than in summer. The amplitude of the first annual harmonic of the event rate has been estimated as $(5.2 \pm 0.1) \%$ with the maximal intensity in the middle of January, and the minimal one in July. Thus, the difference between the average intensity of muon bundles recorded in winter and in summer exceeds $10 \%$. Taking into account that the mean energy of muons registered in the bundles is of the order of several tens $\mathrm{GeV}$, the observed difference cannot be explained in frame of a well-known mechanism of the formation of the temperature effect due to decays of low energy particles in the atmosphere, which is typical for single muons detected at the ground level. An alternative explanation related with changes of the shape of the lateral distribution function of EAS muons in the atmosphere with a variable temperature profile is discussed.
\end{abstract}

The 34th International Cosmic Ray Conference

30 July - 6 August, 2015

The Hague, The Netherlands

\footnotetext{
* Speaker. 


\section{Introduction}

The rate of the events generated as a result of interactions of cosmic rays in the atmosphere and detected on the Earth's surface is subject to variations caused by atmospheric conditions (atmospheric pressure; altitude distribution of temperature and, respectively, air density; water content, etc.). The best studied are variations of the intensity of single cosmic ray muons [1]. The increase of atmospheric pressure leads to an increase of the matter thickness above the observation point and hence to the decrease of muon intensity due to the absorption of less energetic particles in the atmosphere. This phenomenon is referred to as the barometric effect and may be approximately described by the introduction of the so-called barometric coefficient. For low threshold energy of muons, the value of the barometric coefficient is about 0.3 $\% / \mathrm{mm} \mathrm{Hg}$ (negative in sign); with the increase of the threshold energy, the value of the barometric coefficient rapidly decreases. The temperature effect in the muon intensity is related, in the first turn, with the increase of the probability of decay of low energy muons because of the increase of their geometrical path from the generation point to the observation level when the atmosphere is heated. The value of the temperature coefficient (also negative in sign) is about $0.2 \% / \mathrm{K}$. With the increase of the threshold muon energy the value of the temperature coefficient for the total muon intensity decreases, and at energies of the order of tens $\mathrm{GeV}$ changes its sign because of the inclusion of the competitive mechanism related with the increase of the decay probability of parent particles in a warmed (rarified) atmosphere. The temperature effect is the main source of seasonal variations in the intensity of single muons. It is important to note however that the values of meteorological effects as well as the physical processes responsible for their formation are different for different cosmic ray components (muon, hadronic, electron-photon) and events of different classes. The correct understanding of the influence of atmospheric effects on the intensity of registered events is important for accurate comparison of the data of experiments conducted in different conditions and introduction of necessary corrections.

In the present paper, seasonal variations and meteorological effects (barometric and temperature ones) in the intensity of muon bundles detected at the ground level are considered. The bundle is an event with a simultaneous passage of several genetically related muons through the setup. The main source of muon bundles are decays of pions and kaons produced in a nuclear cascade initiated by a high-energy primary cosmic ray particle. Already in the first series of experiments on detection of muon bundles with the coordinate detector DECOR conducted in 2004 - 2007, significant changes of the rate of registered events (far beyond the statistical uncertainties) were noticed [2]. The analysis showed that these changes correlate with the changes of meteorological conditions (atmospheric pressure and ground level air temperature). For the first time, experimental estimates of the barometric and temperature coefficients for the muon bundle intensity were obtained. The value of the temperature coefficient (negative in sign) appeared about two times higher $(\sim 0.5 \% / \mathrm{K})$ than for single muons. Since the mean energy of muons detected in the bundles is approximately by the order of magnitude higher than that of single muons, it is impossible to explain these variations in frame of a usual mechanism of low-energy muon decays. As a possible interpretation, an alternative mechanism related with the geometrical transformation of the lateral distribution function of EAS muons for a varying temperature profile of the atmosphere was suggested. 
If the scenario suggested in [2] for the explanation of the observed value of the temperature effect is correct, it must lead to pronounced seasonal variations in the intensity of muon bundles. Unfortunately, preceding series of measurements with DECOR did not exceed 67 months, and no data were taken in the periods from July to September. Starting from 2012, nearly continuous measurements at the NEVOD experimental complex are conducted, which creates necessary pre-requisites for the analysis of seasonal variations of various types of events on a qualitatively new level. In the present paper, the data on the intensity of muon bundles detected with the coordinate-tracking detector DECOR during three years of observations (2012 $-2015)$ are analyzed.

\section{Experimental setup and the data}

The coordinate-tracking detector DECOR [3] is the part of the NEVOD experimental complex. It is deployed in the galleries of the building of the complex in MEPhI (Moscow, 55. $7^{\circ}$ $\mathrm{N}, 37.7^{\circ} \mathrm{E}$ ) and consists of eight assemblies (supermodules, SMs), each with area of $8.4 \mathrm{~m}^{2}$. Every supermodule includes 8 vertical planes of plastic streamer tube chambers equipped with two-coordinate external strip readout system. The supermodule provides a high spatial and angular accuracy of muon track reconstruction (better than $1 \mathrm{~cm}$ and $1^{\circ}$, respectively).

The selection of muon bundles in DECOR is based on the assumption that the tracks of muons generated in the atmosphere at large distances from the setup are nearly parallel to each other. At a trigger level, the events with at least three of eight simultaneously (within the $250 \mathrm{~ns}$ time gate) actuated SMs of the coordinate detector are selected and registered. The trigger rate for such events is about 0.25 per second. Experimental data are accumulated as a sequence of separate data sets (runs) with the duration up to $\sim 40 \mathrm{~h}$ of live time of observations. At the offline data processing, the geometry reconstruction of tracks in each SM is performed, and candidate events with at least three quasi-parallel tracks (coinciding in direction within $5^{\circ}$ cone) detected in three different SMs of DECOR are selected. A visual scanning of a sample of such events showed that approximately $94 \%$ of them really contain groups of quasi-parallel particles and satisfy the conditions of muon bundle selection. The main admixture (about $6 \pm 1 \%$ ) is constituted by the events related with the detection of electron-photon component of low-energy extensive air showers and with program reconstruction of faked tracks in them. The mean zenith angle of the selected muon bundles is close to $40^{\circ}$. According to the available estimates [4], these bundles are mainly formed as a result of interactions of primary cosmic ray particles with typical energies of $10^{15}-10^{16} \mathrm{eV}$.

Data accumulated in long-term measurements over the period from May 03, 2012 to April 08, 2015 have been used in the present analysis. Short runs with the duration less than $10 \mathrm{~h}$ live time have been excluded from the consideration; altogether, these short runs contain less than $3 \%$ of the data. As a result, we left with 590 runs corresponding to the total time of 17,760 h, containing about 4.4 million muon bundle candidate events. For every run, the number of selected events, the live time of registration, the moments of run beginning and completion (start, stop), average value of the atmospheric pressure at the setup location, and other parameters have been determined. The mean rate of muon bundle events over the period of measurements equals to 248.1 per hour; the average value of the atmospheric pressure is equal to $746.7 \mathrm{~mm} \mathrm{Hg}$. 


\section{Seasonal variations of the muon bundle intensity}

The measured time dependence of the rate of the muon bundle detection over the whole considered period of observations is presented by the points in Fig. 1 (the error bars indicate the statistical uncertainties).

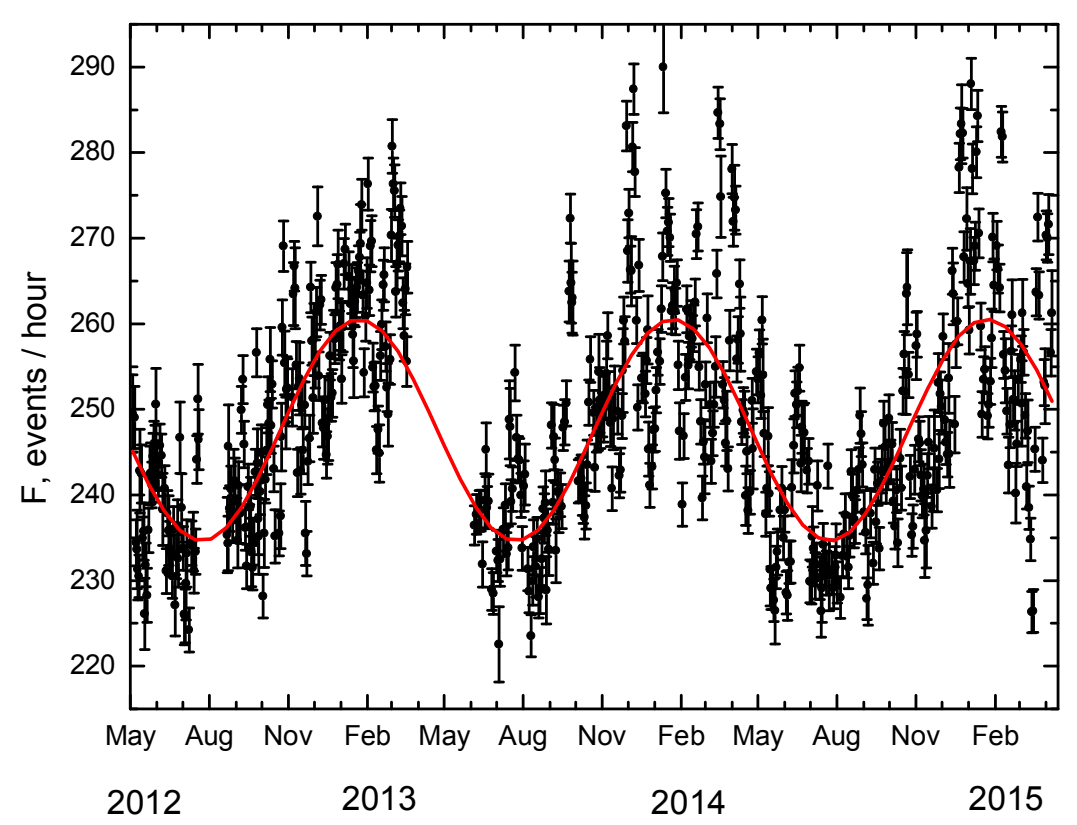

Figure 1: Temporal variation of the muon bundle registration rate during the period of measurements. Points: present experiment (every point represents one run data); smooth curve: the first annual harmonic.

As it follows from the figure, clear seasonal variations repeated every year are observed in the event rate. In winter months, the rate of muon bundle registration is significantly higher than in summer. The red curve in the figure represents the results of the fitting of experimental points with a harmonic function in the form

$$
F\left(t_{i}\right)=C+A \cos \left(2 \pi\left(t_{i}-t_{\mathrm{m}}\right) / t_{0}\right)
$$

where $t_{i}$ is the time (days from the beginning of 2012) corresponding to the middle of the $i$-th data set (run), $t_{0}$ is the period equal to the mean calendar year (365.24 days); $t_{\mathrm{m}}$ is the time moment in the year corresponding to the maximum of the first annual harmonic. As a result of the application of the weighted least squares technique, the following values of the parameters have been found: $C=247.58 \pm 0.12$ events $/ \mathrm{h} ; A=12.94 \pm 0.17$ events $/ \mathrm{h} ; t_{\mathrm{m}}=21.4 \pm 0.8$ day (errors are pure statistical). Thus, on the average, the intensity of muon bundles detected in January and July differs by more than $10 \%$; the maximum intensity is typically observed in the second half of January, and the minimal one in the middle of July.

\section{Barometric and temperature effects}

On the background of smooth seasonal variations in the intensity of the detected events during the year (Fig. 1), short-term deviations from the harmonic dependence with a duration of the order of few days are observed, for example, increases of the event rate in the end of September and in December 2013, in January and in June 2014, etc. It is naturally to assume 
that these deviations are related with short-term changes of the state of the atmosphere caused by the active processes in it. We have performed the analysis of correlations of muon bundle intensity with the atmospheric pressure and the mass average temperature of atmospheric air near the point of the location of the setup. As a source of information about the temperature of the atmosphere, we have used the retrospective data of the GDAS model (Global Data Assimilation System) [5], in the database of which, in particular, the values of the air temperature for a set of fixed levels of the residual atmospheric pressure are presented. Threehour data of the GDAS model for the nearest knot of the geographical grid $\left(56^{\circ} \mathrm{N}, 38^{\circ} \mathrm{E}\right)$ are used. For the comparison with the data on muon bundle intensity, we have defined the mass average temperature of the atmospheric air as

$$
T_{\mathrm{ma}}=\int_{P_{1}}^{P_{2}} T(P) d P / \int_{P_{1}}^{P_{2}} d P,
$$

where $T(P)$ is the air temperature at the pressure level $P$, and as integration limits $P_{1}$ and $P_{2}$ the values 10 and 950 mbar have been chosen. After that, for every set of data with muon bundles, the value of the mass average temperature was estimated as a simple average of 3-hour GDAS data within the start/stop limits of the run. The mean value of the mass average temperature of atmospheric air for the whole period of the experiment $\left\langle T_{\mathrm{ma}}>=247.0 \mathrm{~K}\right.$.

In Fig. 2 and Fig. 3, correlations of the rate of muon bundle detection respectively with the atmospheric pressure and the mass average air temperature are presented. The lines in the figures show the results of linear regression taking into account the statistical weights of individual points (runs).

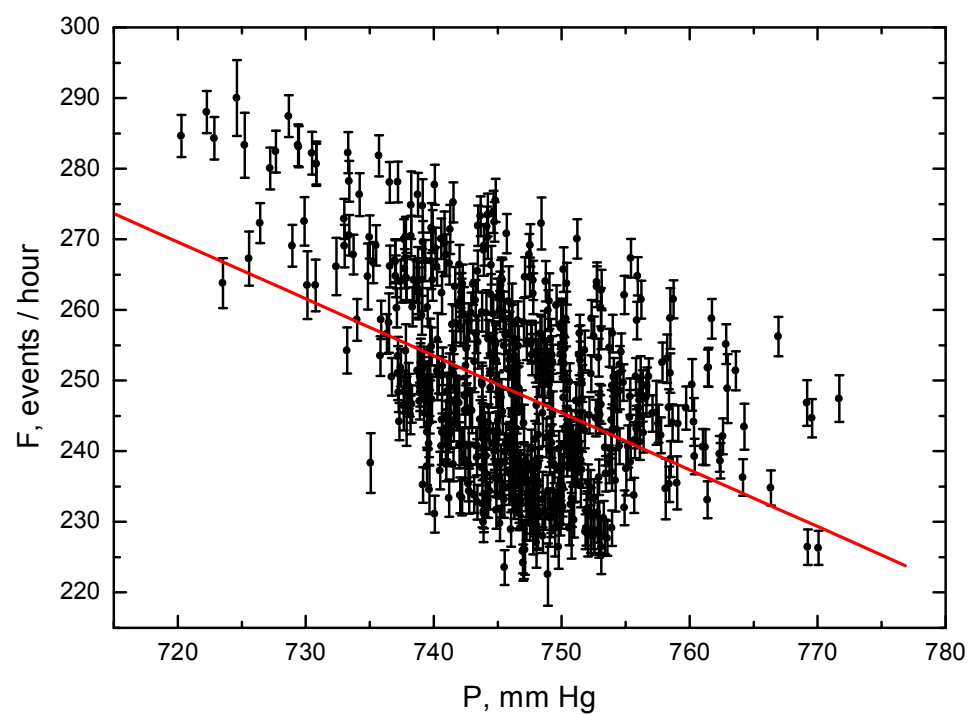

Figure 2: Correlations of the rate of muon bundle detection with the atmospheric pressure at the observation point. The line: linear regression.

The slope of the regression line for the dependence on the atmospheric pressure (Fig. 2) corresponds to the value of the barometric coefficient $\beta_{P}=-(0.325 \pm 0.006) \% / \mathrm{mm} \mathrm{Hg}$. At the same time, a large spread of the experimental points relative to the regression line is seen (the value of the corresponding correlation coefficient $R$ equals to -0.45 ), which indicates a strong influence of other factors that affect the muon bundle intensity (first of all, the changing temperature profile of the atmosphere). 


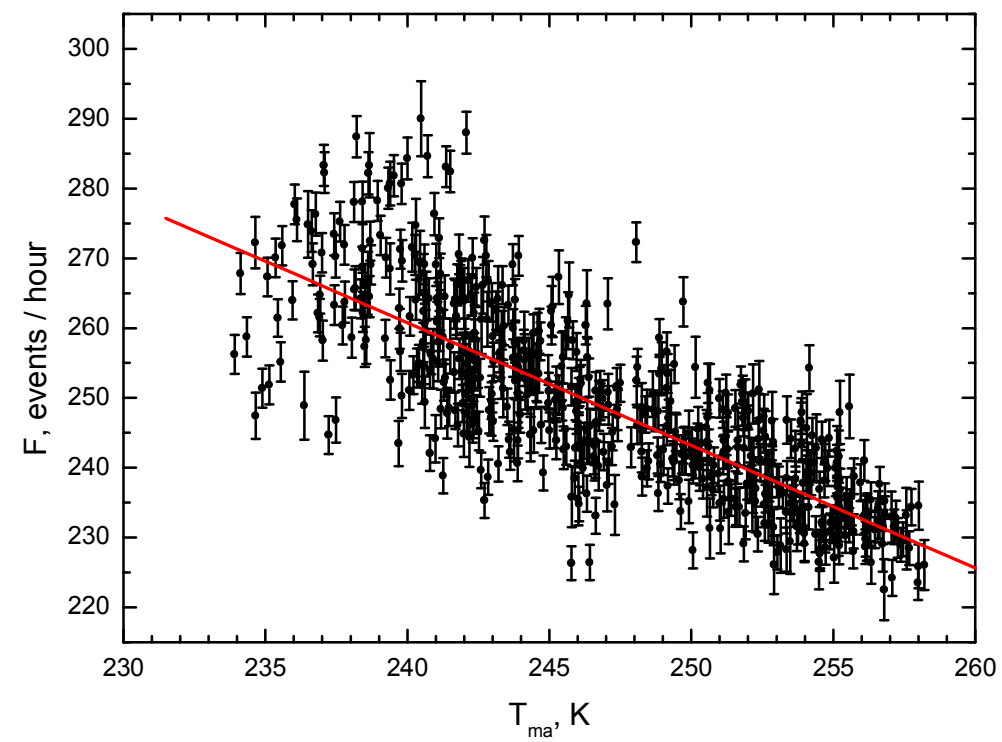

Figure 3: Correlations of the muon bundle detection rate with the mass average temperature of the atmospheric air in the vicinity of the observation point.

Much more close correlations are observed in the comparison of the muon bundle detection rate with the mass average air temperature (Fig. 3, correlation coefficient $R=-0.80$ ), which evidences that the temperature effect is the main factor that influences the intensity of the events. The estimate of the temperature coefficient on the basis of linear regression of the data presented in Fig. 3 is $\beta_{T}=-(0.708 \pm 0.008) \% / \mathrm{K}$ (the error is statistical).

In the paper [2], a simple geometrical mechanism of the formation of the temperature effect for muon bundles was suggested. Detection of muon bundles at the surface corresponds to the selection of events (EAS muon component) according to value of the local muon density at the observation point [4]. Muons which form the bundles are produced in decays of pions and kaons generated near the extensive air shower core. Their geometrical spread on the surface is mainly determined by the transverse momenta of parent hadrons at production and the geometrical altitude of their generation. In its turn, for any fixed depth $X\left(\mathrm{~g} \mathrm{~cm}^{-2}\right)$ in the atmosphere the altitude is proportional to the absolute atmosphere temperature. Thus, the changes in the air temperature lead to the changes of the typical spread of muons at the level of observations and to a modification of the lateral distribution function of EAS muons (see Fig. 4 as an illustration). As shown in [2], under these assumptions the intensity of muon bundles should be proportional to a power function of the effective atmosphere temperature:

$$
F(T) \sim(<T>/ T)^{2(\beta-1)},
$$

where $\beta=(1.90 \pm 0.02)$ is the power index of the integral spectrum of events in local muon density in a relevant range of particle densities and zenith angles [4]. Then, differentiating the equation (3), one can find the temperature coefficient for the muon bundle intensity near the effective temperature $<T>$ as

$$
\frac{1}{F} \frac{d F}{d T}=-\frac{2(\beta-1)}{<T>} .
$$

If we take as an estimate of the effective air temperature the mean value of the mass average temperature over the observation period $(247 \mathrm{~K}$, see above), we will readily obtain a 
quantitative estimate of the expected temperature coefficient due to the considered geometrical mechanism: $\beta_{T}=-(0.77 \pm 0.02) \% / \mathrm{K}$, which is in a quite good agreement with the value obtained experimentally.
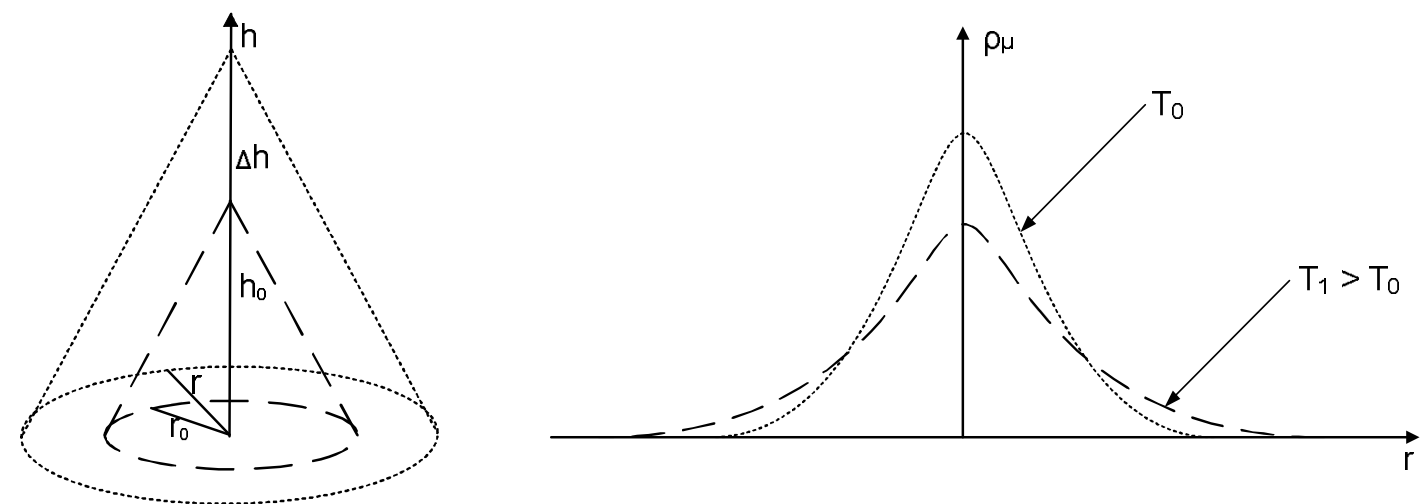

Figure 4: Illustration of the increase of the radial spread of muons (left) and of changes of the lateral distribution function of EAS muons (right) at the heating of the atmosphere [2].

\section{Conclusion}

The analysis of the data of a three-year experiment on detection of muon bundles at the Earth's surface conducted with the coordinate-tracking detector DECOR has revealed the presence of clear seasonal variations in the event rate. The difference in the muon bundle intensity in winter and summer months exceeds $10 \%$. Comparison of the measured event rate with atmospheric pressure and mass average air temperature during the observation period has shown that the main factor that influences the muon bundle intensity is the temperature effect. The experimental value of the temperature coefficient is in a good agreement with calculations performed in frame of a simple geometrical mechanism of the formation of the temperature effect for the muon bundle intensity, related with the changes of the lateral distribution function of EAS muons at the observation level caused by the shift of the particle generation altitudes at the varying temperature of the atmosphere.

The work was performed at the Unique Scientific Facility "Experimental complex NEVOD". It was supported by the Ministry of Education and Science of the Russian Federation (project RFMEFI59114X0002, the government task and the program for Leading Scientific Schools, project NSh-4930.2014.2).

\section{References}

[1] L.I. Dorman. Meteorological effects in cosmic rays. Moscow: Nauka (1972).

[2] N.V. Tolkacheva et al., Bull. Russ. Acad. Sci. Physics, 75, 377 (2011).

[3] N.S. Barbashina et al., Instrum. Experim. Techniques, 43, 743 (2000).

[4] A.G. Bogdanov et al., Phys. Atom. Nucl., 73, 1852 (2010).

[5] NOAA Air Resources Laboratory (ARL), http:/ready.arl.noaa.gov/gdas1.php 